\title{
Polyelectrolyte Solutions with Multivalent Salts
}

Paulo S. Kuhn (1), Yan Levin (1) *, and Marcia C. Barbosa (1,2)

(1) Instituto de Física, Universidade Federal do Rio Grande do Sul Caixa Postal 15051, CEP 91501-970, Porto Alegre, RS, Brazil

(2) Institute for Theoretical Physics

University of California, Santa Barbara, CA, 93106-2431, USA

e-mails: kuhn@if.ufrgs.br, levin@if.ufrgs.br,

barbosa@if.ufrgs.br, barbosa@itp.ucsb.edu

\begin{abstract}
We investigate the thermodynamic properties of a polyelectrolyte solution in a presence of multivalent salts. The polyions are modeled as rigid cylinders with the charge distributed uniformly along the major axis. The solution, besides the polyions, contain monovalent and divalent counterions as well as monovalent coions. The strong electrostatic attraction existing between the polyions and the counterions results in formation of clusters consisting of one polyion and a number of associated monovalent and divalent counterions. The theory presented in the paper allows us to explicitly construct the Helmholtz free energy of a polyelectrolyte solution. The characteristic cluster size, as well as any other thermodynamic property can then be determined by an appropriate operation on the free energy.
\end{abstract}

\section{Introduction}

The polyelectrolytes are ubiquitous in our every day life. They are present in a variety of industrial products ranging from the hair conditioners to the superabsorbants used in baby diapers. The carrier of genetic code, the DNA, is also a polyelectrolyte. Not withstanding their omnipresence, our understanding of this class of polymers is far from complete. The long range Coulomb interaction combined with a strong asymmetry existing between the various entities of a polyelectrolyte solution, has proven to be a formidable challenge to the standard methods of liquid state theory. The scaling approaches which were so successful in elucidating the properties of non-ionic polymers have, so far, failed in their extension to polyelectrolytes. What seems to be lacking is a meanfield theory similar to the one constructed by Flory for the simple polymers and by Debye and Hückel for the symmetric electrolytes. Recently, we have attempted to construct such a mean-field theory for the special class of rigid polyelectrolytes, an example of which is provided by a solution of DNA segments. The advantage of working with a rod-like polyelectrolytes is that the intramolecular degrees of freedom associated with the conformational state of a polyion can be neglected. The polyelectrolyte solution then becomes a strongly asymmetric electrolyte, to which a generalization of the Debye-Hückel-Bjerrum (DHBj) theory [1] can be applied. Additional advantage in working with rigid polyelectrolytes is that they obey the Manning infinite dilution limiting laws [2]. These laws are extremely useful since they provide the bound- ary conditions that any theory of rod-like polyelectrolytes must satisfy.

We have demonstrated that the extension of the DHBj theory to rigid polyelectrolytes, indeed, satisfies the Manning Limiting Laws [3]. Furthermore, the theory is in excellent agreement with the experiments on rigid polyelectrolyte solutions in a presence of monovalent salt 泊. Recently we have extended our work to study the interaction of a polyelectrolyte with a cationic surfactants [0. The latter problem is of great practical interest in a variety of biochemical and biomedical applications related to design of gene delivery systems [6]. Again, the DHBj theory has proven successful in giving quantitative agreement with experiments without a need for any adjustable parameters. In the current work we shall extend the theory to allow for the presence of both the monovalent and the divalent salts. This problem, once again, is motivated by the biomedical applications to gene therapy. It has been recently found that introduction of a DNA into host cells is facilitated by the presence of a small amount of divalent salt such as calcium phosphate [6.

Since the bulk of the calculations is similar to those presented in the earlier work, we shall not enter in great detail and instead refer the reader to the previously published papers $[3$ 5].

${ }^{*}$ Corresponding author 


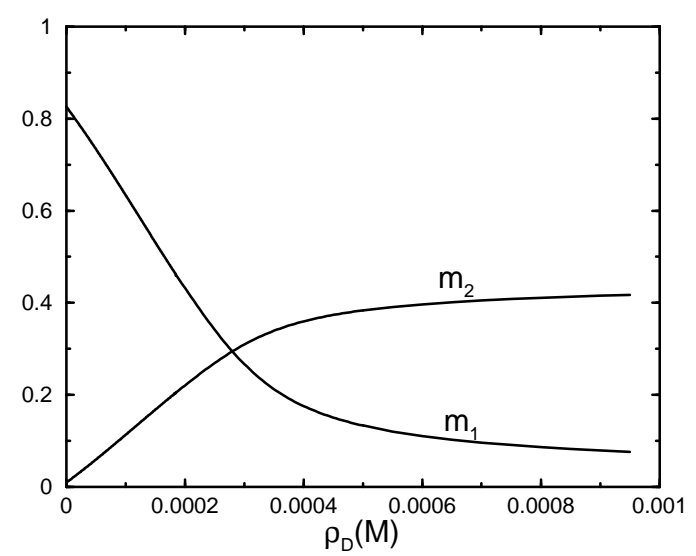

FIG. 1. The DNA binding isotherms for the monovalent and divalent counterions. The parameters are: $Z=440$, $\xi=4.17, \rho_{p}=2 \times 10^{-6} \mathrm{M}, \rho_{M}=10^{-2} \mathrm{M}, a_{p}=27 \AA$, $a_{c}=7.04 \AA$.

\section{The model}

We begin by defining the Primitive Model of Polyelectrolyte (PMP) [3]. Our system consist of long cylindric polyions, spherical counterions, monovalent salt, and divalent salt, inside the volume $V$. The polyions, of length $L$, have diameter $a_{p}$ and charge $-Z q$ uniformly distributed along the length of the cylinder. The distance between the charged groups is $b \equiv L / Z$. The counterions and coions are modeled as rigid spheres of diameter $a_{c}$ and charge $+q,+2 q,-q$ located at their centers. We shall not distinguish between the monovalent counterions derived from the polymers or salt, and assume that all the salt is dissociated. Similarly, to simplify calculations, we shall treat the coions derived from the monovalent and the divalent salts as being identical. The solvent is modeled as a continuum medium of dielectric constant $D$. The distance of closest approach, $a$, between a polyion and a counterion is $a=\left(a_{p}+a_{c}\right) / 2$. This represents the "exclusion cylinder" due to the hard-core repulsion.

The strong electrostatic attraction existing between the macromolecules and the counterions results in some number of counterions becoming associated with the polyions, producing clusters composed of one polyion, $m_{B}$ monovalent counterions, and $d_{B}$ divalent counterions. In fact, we expect that there shall be a distribution of cluster sizes. Motivated, however, by our previous work [4] we know that the polydispersity in cluster sizes is not very important, if one is interested in thermodynamics of a polyelectrolyte solution [7]. Since not all of the counterions condense onto the polyions, for any nonzero temperature some free, unassociated, counterions remain in the solution. We denote the density of unassociated monovalent counterions as $\rho_{+}=\left(Z-m_{B}\right) \rho_{p}+\rho_{M}$, and the density of unassociated divalent counterions as $\rho_{++}=\rho_{D}-d_{B} \rho_{p}$, where $\rho_{p}, \rho_{M}$, and $\rho_{D}$ are the densities of the polyions, the monovalent salt, and the divalent salt respectively. Since the coions do not participate in association their density remains unchanged, $\rho_{-}=\rho_{M}+2 \rho_{D}$. The goal of the theory is to determine the characteristic cluster size, i.e. the values of $m_{B}$ and $d_{B}$.

Complete thermodynamic information about the system is contained in its Helmholtz free energy. The condition that the free energy must be minimum allows us to determine the values of $m_{B}$ and $d_{B}$. Once this is done, all the thermodynamic functions of the system can be found through the appropriate operations on the free energy. For example, the pressure inside the polyelectrolyte solution is a Legendre transform of the Helmholtz free energy density $f=-F / V$, given by $p\left(T,\left\{\rho_{t}\right\}\right)=f\left(T,\left\{\rho_{t}\right\}\right)+\sum \mu_{t} \rho_{t}$, where the chemical potential of a specie of type $t$ (clusters, counterions, and coions) is $\mu_{t}=-\partial f / \partial \rho_{t}$.

The free energy cannot be calculated exactly. We shall, therefore, attempt to construct it as a sum of the most relevant contributions. These can be divided into an electrostatic and an entropic ones. The electrostatic contribution arises as the result of polyion-counterion, polyion-polyion, and the counterion-coion interactions. The entropic contribution is the result of mixing of various species [3].

The free energy for the polyion-counterion interaction is calculated in the framework of the Debye-Hückel (DH) theory [8]. The full calculation is presented in Ref. [4] and here we shall just quote the result,

$$
\begin{aligned}
\beta f^{p c}= & \rho_{p}\left(Z-m_{B}-2 d_{B}\right)^{2} \frac{(a / L)}{T^{*}(\kappa a)^{2}} \times \\
& \times\left\{2 \ln \left[\kappa a K_{1}(\kappa a)\right]-I(\kappa a)+\frac{(\kappa a)^{2}}{2}\right\}
\end{aligned}
$$

with $\left.\kappa a=\sqrt{(} 4 \pi \rho_{1}^{*} / T^{*}\right), T^{*}=D k_{B} T a / q^{2}$ is the reduced temperature, $\rho_{1}^{*}=\rho_{1} a^{3}$, and

$$
I(\kappa a) \equiv \int_{0}^{\kappa a} d x \frac{x K_{0}^{2}(x)}{K_{1}^{2}(x)} .
$$

where $K_{n}$ is the Bessel function of order $n$. The modification of replacing the total microion density by twice the ionic strength, $\rho_{1} \equiv \rho_{+}+4 \rho_{++}+\rho_{-}$, is necessary when discussing multivalent salts.

The contribution to the free energy due to the interactions among the various free ions is given by the usual DH theory 8,

$$
\beta f^{c c}=\frac{1}{4 \pi} \frac{1}{a_{c}^{3}}\left\{\ln \left(1+\kappa a_{c}\right)-\kappa a_{c}+\frac{\left(\kappa a_{c}\right)^{2}}{2}\right\} .
$$

The free, unassociated, counterions and coions screen the polyion-polyion interactions, producing an effective potential of short range. The polyion-polyion contribution to the free energy can then be calculated using a 
Van der Waals type of approach [4]. To this end the polyion-polyion contribution is expressed as a second virial term, averaged over the relative angle sustained by two molecules. The result is,

$$
\beta f^{p p}=\frac{-2 \pi a^{3} \exp (-2 \kappa a)\left(Z-m_{B}-2 d_{B}\right)^{2} \rho_{p}^{2}}{T^{*}(\kappa a)^{4} K_{1}(\kappa a)^{2}} .
$$

The entropic contribution is found using the Flory theory [9]. Thus, the increase in free energy due to mixing of various species is the sum of their ideal free energies,

$$
\beta f^{e n t}=\sum_{t}\left[\rho_{t}-\rho_{t} \ln \left(\phi_{t} / \zeta_{t}\right)\right]
$$

where, in obvious notation, the respective volume fractions are,

$$
\begin{aligned}
& \phi_{c l}=\frac{\pi \rho_{p}^{*}}{4(a / L)}\left(\frac{a_{p}}{a}\right)^{2}+\left(m_{B}+d_{B}\right) \frac{\pi \rho_{p}^{*}}{6}\left(\frac{a_{c}}{a}\right)^{3}, \\
& \phi_{+}=\frac{\pi \rho_{+}^{*}}{6}\left(\frac{a_{c}}{a}\right)^{3}, \\
& \phi_{++}=\frac{\pi \rho_{++}^{*}}{6}\left(\frac{a_{c}}{a}\right)^{3}, \\
& \phi_{-}=\frac{\pi \rho_{-}^{*}}{6}\left(\frac{a_{c}}{a}\right)^{3} .
\end{aligned}
$$

$\zeta_{t}$ is the internal partition function of an isolated specie $t$. For structureless particles, $\zeta_{+}=\zeta_{-}=\zeta_{++} \equiv 1$. The calculation of $\zeta_{c l}$, proceeds along the lines given in Ref. [4, 5]. We find that to a good accuracy the internal partition function of a cluster can be approximated by

$$
\begin{aligned}
\ln \zeta_{c l}= & -\xi S\left(-2 m_{1}-4 m_{2}+4 m_{1} m_{2}+m_{1}^{2}+4 m_{2}^{2}\right)+ \\
& -Z\left[\left(1-m_{1}-m_{2}\right) \ln \left(1-m_{1}-m_{2}\right)+\right. \\
& \left.+m_{1} \ln m_{1}+m_{2} \ln m_{2}\right]
\end{aligned}
$$

where the binding fractions are, $m_{1}=m_{B} / Z, m_{2}=$ $d_{B} / Z$, and $S$ is

$$
S \equiv \frac{1}{2} \sum_{s_{1} \neq s_{2}} \frac{1}{\left|s_{1}-s_{2}\right|}=Z[\psi(Z)-\psi(1)]-Z+1
$$

where $\psi(n)$ is the digamma function and $\xi \equiv \beta q^{2} / D b$ is the Manning parameter [2].

\section{Results and Conclusions}

Adding the above contributions we obtain the full Helmholtz free energy for the polyelectrolyte plus salt system, $f=f^{e n t}+f^{p c}+f^{c c}+f^{p p}$. Minimization of the free energy allows us to find the number of condensed monovalent and divalent counterions, $m_{B}$ and $d_{B}$. In Fig.1 we show the dependence of the binding fractions on the density of divalent salt, at fixed polyion and monovalent salt concentrations. At low densities of divalent salt, the condensation is dominated by the monovalent ions, since the divalent ions gain more entropy, and thus lower the total free energy, by staying free. However as the concentration of divalent salt increases, the gain in electrostatic energy due to condensation wins over the entropy and more divalent ions become associated with the macromolecules. We note that unlike the association with the ionic surfactants, which exhibits a large degree of cooperativity characterized by the sharp rise in the surfactant binding fraction, the replacement of the condensed monovalent counterions by the divalent counterions proceeds quite smoothly.

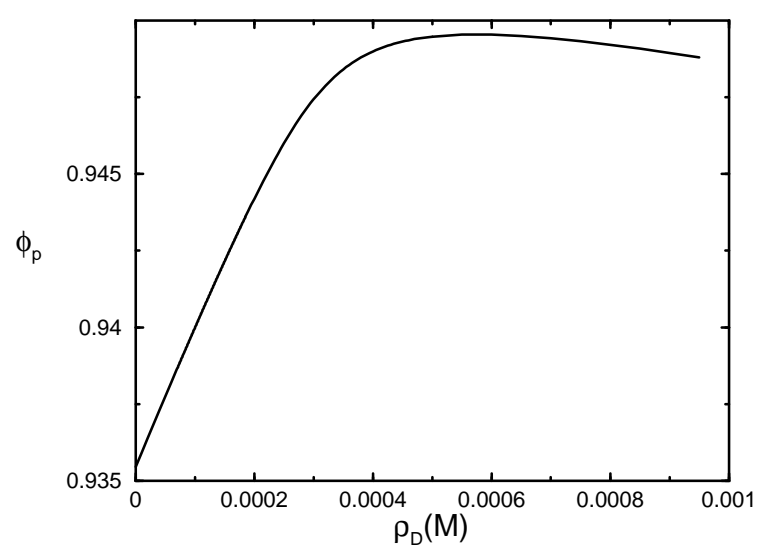

FIG. 2. The osmotic pressure coefficient for the polyelectrolyte multivalent salt system. The parameters have the same value as in Fig.1.

This result could have been anticipated a priori. The presence of a condensed divalent counterion does not stimulate any further condensation of additional particles, but on the contrary, the electrostatic repulsion between the like-charged counterions tends to inhibit any further association. The situation is quite different in the case of binding by the ionic surfactants. After the first amphiphile is associated, the condensation of additional molecules is energetically favored since the buildup of the hydrocarbon density in the vicinity of a polyion helps to exclude water and, thus, reduces the unfavorable hydrophobic energy of the alkyl tails. This, then, explains why the association with ionic surfactant and lipids exhibits a large degree of cooperativity while the binding of divalent counterions proceeds in a completely uncooperative fashion.

Finally, using the number of associated ions, the osmotic pressure, defined as $\beta p \equiv\left(Z \rho_{p}+2 \rho_{M}+3 \rho_{D}\right) \phi_{p}$, can be computed as a function of divalent salt density (Fig. 2). 


\section{ACKNOWLEDGMENTS}

This work was supported in part by CNPq - Conselho Nacional de Desenvolvimento Científico e Tecnológico and FINEP - Financiadora de Estudos e Projetos, Brazil. This research was also supported by the National Science Foundation under Grant No. PHY94-07194.

[1] M. E. Fisher and Y. Levin, Phys. Rev. Lett. 71, 3826 (1993); Y.Levin and M. E. Fisher, Physica A 225, 164 (1996).

[2] G.S. Manning, Quart. Rev. of Biophys. II 2, 179 (1978);
J.L. Barrat and J. F. Joanny, Adv. Chem. Phys. 94, 1, (1996); G. S. Manning, J. Chem. Phys. 51, 924 (1969).

[3] Y. Levin, Europhys. Lett. 34, 405 (1996); Y. Levin and M. C. Barbosa, J. Phys. II (France) 7, 37 (1997).

[4] P. S. Kuhn, Y. Levin, and M. C. Barbosa, Macromolecules (in press).

[5] P. S. Kuhn, Y. Levin, and M. C. Barbosa, "Complex Formation Between Polyelectrolyte and Ionic Surfactant" con-mat/9806226

[6] P. L. Felgner and G. M. Ringold, Nature 337, 387 (1989)

[7] Y. Levin, M. C. Barbosa, and M. N. Tamashiro Europhys. Lett. 41, 123 (1998)

[8] (a) P.W. Debye and E. Hückel, Phys. Z. 24, 185 (1923); (b) For a good exposition see D.A. McQuarrie, Statistical Mechanics (Harper and Row, New York, 1976), Chap. 15.

[9] P. Flory, Principles of Polymer Chemistry (Cornell University Press, Ithaca, New York, 1971). 